\title{
Una experiencia de trabajo interdisciplinario en dolor crónico. Resultados y desafíos
}

Ma. del Carmen Abreu*, Ma. José Montesł, Gustavo Quinteros ${ }^{\ddagger}$, Jimena Retamoso§, Irene Retamoso๙

\section{Resumen}

El dolor crónico constituye un problema de salud pública a nivel mundial, se estima que afecta alrededor del $20 \%$ de la población. Cada vez hay más evidencia de que el modelo de atención a estos pacientes es a través de equipos interdisciplinarios, con énfasis en la comunicación entre las diferentes disciplinas, y la construcción de una mirada conjunta del paciente.

La Unidad Interdisciplinaria de Dolor del Hospital Maciel - Banco de Seguros del Estado es la puesta en práctica de un modelo biopsicosocial de atención al dolor crónico, donde el objetivo terapéutico es la rehabilitación integral de la persona. En este artículo se describe la población asistida en los primeros siete meses de trabajo en el Banco de Seguros del Estado, la evaluación integral que realiza el equipo y los resultados más relevantes, así como la construcción del plan terapéutico individualizado.

La lumbalgia crónica poscirugía fue el cuadro clínico más frecuente. De la evaluación integral se destaca la interferencia del dolor con las actividades básicas de la vida diaria en $90 \%$, alteraciones del sueño en $60 \%$ y el diagnóstico de depresión en el $40 \%$ de la población asistida. Se detallan las intervenciones realizadas en los tres pilares terapéuticos: farmacológico, rehabilitación física y abordaje psicoterapéutico, con el objetivo definido de la rehabilitación de la persona y su reintegro al trabajo.

Palabras clave: Dolor

Dimensión del dolor

Unidad interdisciplinaria de dolor

Key words: $\quad$ Pain

Pain measurement

Interdisciplinary pain unit

\footnotetext{
* Licenciada en Psicología. Colaboradora especializada del Departamento de Psicología Médica. Ex Prof. Adjunto de Psicología Médica.

† Médico especialista en Medicina Interna y en Tratamiento del Dolor y Cuidados Paliativos. Ex Asistente de Clínica Médica.

‡ Médico especialista en Medicina Interna y en Psiquiatría. Ex Asistente de Clínica Médica y de Psicología Médica.

$\S$ Licenciada en Fisioterapia. Licenciada en Educación Física.

II Médico especialista en Medicina Interna. Especialista en Tratamiento del Dolor y Cuidados Paliativos del Adulto. Ex Prof. Adj. de Clínica Médi-

ca, Ex Prof. Adj. de Emergencia.

Correspondencia: Dra. Irene Retamoso. Llambí 1508. Montevideo, Uruguay. Correo electrónico: retamosoirene@gmail.com

Los autores declaran no tener conflictos de intereses.

Recibido: 29/8/18

Aprobado: 15/11/18
} 


\section{Introducción}

La Unidad Interdisciplinaria de Dolor es la puesta en práctica, en forma conjunta entre el Hospital Maciel y el Banco de Seguros del Estado, de un proyecto que tiene como objetivo asistir en forma integrada, con un enfoque biopsicosocial, a pacientes con dolor crónico de difícil manejo. El objetivo fundamental de este proyecto no es solamente el alivio del dolor, sino la rehabilitación integral del sujeto con este problema de salud.

El dolor crónico constituye un problema de salud pública a nivel mundial, se estima que afecta a $20 \%$ de la población ${ }^{(1,2)}$. Más de $60 \%$ de los pacientes que lo sufren, refieren haber experimentado dolor durante más de cinco años ${ }^{(3)}$, con deterioro de la calidad de vida ${ }^{(4)}$.

A diferencia del dolor agudo, que tiene un valor biológico adaptativo de alarma frente a una situación que amenaza la integridad física del sujeto, el dolor crónico es aquel que persiste luego que la causa que lo desencadenó fue resuelta, o no es posible determinar una lesión que claramente lo explique.

La Asociación Mundial para el Estudio del Dolor (IASP) lo define como "una experiencia sensorial y emocional desagradable, asociada con un daño tisular, real o potencial, o descrita en términos de dicho daño ${ }^{\text {"(5) }}$. Esta definición resulta hoy insuficiente para explicar o pretender explicar el dolor crónico. Hoy día es claro que el dolor crónico es un problema de salud que excede a la sola experiencia de dolor y que engloba a toda la persona en sus diferentes dimensiones, física, psíquica, familiar, sociolaboral y espiritual. Otro intento de definición podría ser: "El dolor crónico puede definirse como un estado sensibilizado de percepción del dolor con la impronta genética, afectiva, cultural y adaptativa individual,"(6).

"El dolor no es la actividad inducida por el estímulo nocivo en el receptor de dolor y en las vías nociceptivas, es siempre un estado psicológico, incluso cuando se pueda apreciar con claridad que el dolor tiene una causa física" ${ }^{\text {"(5). }}$.

El modelo de atención biomédico se muestra totalmente insuficiente para el tratamiento de estos pacientes. Actualmente toda la literatura científica sobre el tema plantea que el mejor modelo de atención de estos pacientes es a través de equipos interdisciplinarios ${ }^{(7)}$. Este modelo de atención exige una conceptualización común del problema, un diagnóstico de situación y una terapéutica planteada en conjunto y adaptada a las características de cada paciente.

El abordaje multidisciplinario es la práctica habitual en los servicios de salud; un mismo paciente es asistido por muchos especialistas, pero entre ellos se mantiene un contacto mínimo, muchas veces solo a través de lo registrado en la historia clínica. Los tratamientos son planteados desde la propia disciplina sin tener en cuenta las otras intervenciones, generando multiplicidad de problemas para el paciente, por ejemplo: polifarmacia, repetición de exámenes complementarios y en gran porcentaje insatisfacción con el sistema asistencial. Es fuente, además, de error médico, por ejemplo por interacciones farmacológicas o por no prestar atención a ciertos aspectos que están siendo vistos por otro especialista. El enfoque interdisciplinario ${ }^{(8)}$ pone el acento en la comunicación entre las disciplinas y los profesionales actuantes, se construye una mirada en conjunto sobre el paciente y no solo sobre los síntomas o probable "enfermedad" del sujeto que consulta $^{(9)}$. Cada profesional, desde su saber, aporta al diálogo, generándose una visión amplia, abarcadora e integradora de los diferentes aspectos del sujeto y su dolencia. Esta visión es lo que se ha dado en llamar paradigma biopsicosocial, el cual entre sus conceptualizaciones integra el paradigma de la complejidad, tomando distancia de la causalidad lineal para adoptar la multicausalidad, apoyándose también en la teoría general de los sistemas. Resumiendo, es una mirada sistémica del sujeto y sus problemas. Este diálogo interdisciplinario hace posible la construcción de un nuevo saber, son momentos de trascendencia de la propia disciplina hacia la generación de un momento transdisciplinario que permite una nueva conceptualización sobre un determinado problema. La transdisciplina queda así entendida como ese estadio superior del saber en que se logra la integración de varios saberes producto de un adecuado y fecundo diálogo interdisciplinario ${ }^{(10)}$.

Diríamos que en el dolor crónico es este el abordaje fundamental, tanto la literatura como la propia experiencia clínica nos ha mostrado que este es el camino adecuado para intervenir en este grave y creciente problema de salud pública. Nuestra más reciente experiencia en la Unidad Interdisciplinaria para Tratamiento del Dolor Crónico del Hospital Maciel y del Banco de Seguros del Estado nos ratifica en nuestra posición. Es una experiencia novedosa, que requiere la formación de equipos adecuados, que logren trabajar como verdaderos equipos integrados, que compartan el saber, la experiencia y a través del trabajo en común lograr construir nuevas evidencias. En este sentido, el trabajo clínico asistencial articulado con la investigación es fundamental, apuntando los esfuerzos a lograr una rehabilitación integral del sujeto que lo mantenga integrado a su familia, sociedad y trabajo. Está entre los objetivos reducir el daño por procedimientos innecesarios, así como reducir los costos en el proceso asistencial, al reducir estudios y procedimientos diagnósticos. 


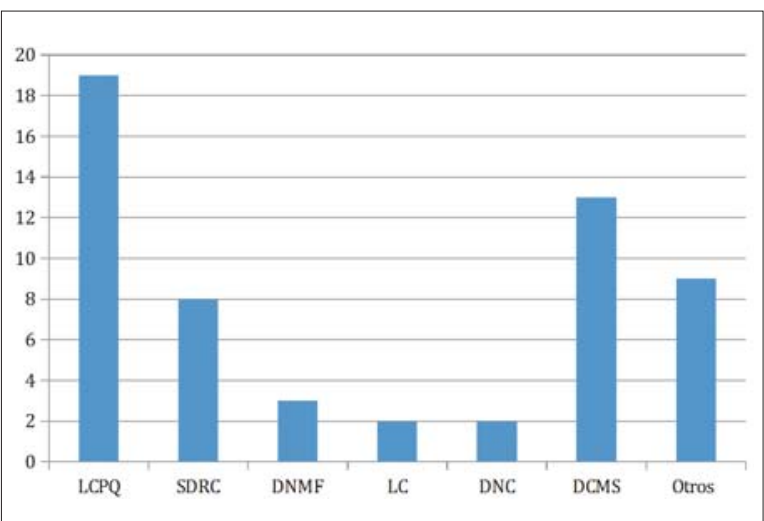

Figura 1. Situaciones clínicas de pacientes asistidos de mayo a diciembre de 2017.

\section{Población asistida en la Unidad Interdisciplinaria de Dolor en el BSE en el período comprendido entre mayo y diciembre de 2017}

En este período asistimos a un total de 73 siniestrados, 43 hombres y 30 mujeres, de entre 23 y 61 años de edad.

El 26\% de la población asistida llevaba entre un año o dos de evolución. El 16,4\% de dos a cinco años, el 26\% más de cinco años (figura 1).

\section{Resultados de la evaluación integral de los pacientes asistidos en el período citado. Aspectos más relevantes}

En la práctica clínica habitual se utilizan diferentes escalas para intentar objetivar la intensidad del dolor ${ }^{(11)}$. Tenemos que tener en claro que el dolor es una percepción subjetiva, por lo cual es imposible de medir. En la Unidad Interdisciplinaria de Dolor utilizamos la escala numérica verbal. La utilidad que podría tener el uso de esta escala es para objetivar la evolución de la percepción que tiene el paciente de su dolor, para lo cual se aplica en la primera consulta y luego en la evolución.

En el sujeto con dolor crónico el objetivo principal es mejorar a la persona, lo cual no es sinónimo de la mejoría del dolor. Muchas veces los pacientes refieren sentirse mejor, funcionalmente comienzan a realizar más actividades, mejoran el descanso, el ánimo, pero la intensidad del dolor se mantiene igual. Pero el paciente está mejor y este es el objetivo de las unidades de dolor crónico (figura 2).

La evaluación integral del paciente con dolor crónico debe incluir, además de la intensidad y todas las características clínicas del dolor, los aspectos personales, familiares y sociales ${ }^{(12)}$. Entre ellos, destacamos: percepción que tiene de su situación, creencias sobre sus síntomas, su enfermedad y los tratamientos realiza$\operatorname{dos}^{(13)}$. La personalidad en la cual se incluyen rasgos, estilos de defensa y afrontamiento, sistemas de creencias,

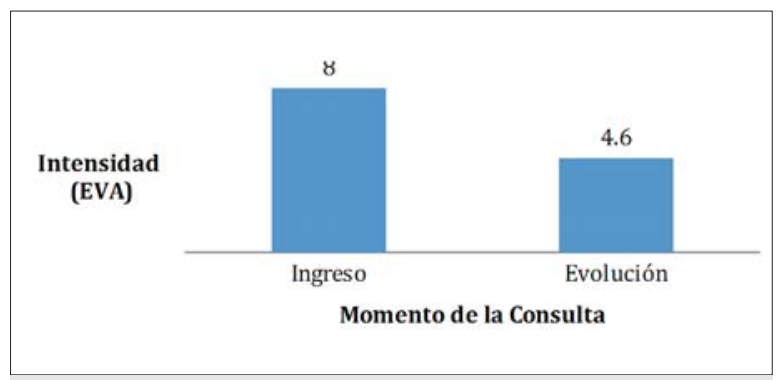

Figura 2. Promedio de intensidad de dolor de los pacientes en la primera consulta y en la consulta de control ( $7 \%$ de los pacientes refirieron alivio sin asignar número)

estilos afectivos y cognitivos, y aspectos conductuales y comportamentales ${ }^{(14,15)}$. La historia familiar, vínculos familiares, situación social y vínculos sociales son aspectos de gran relevancia.

En la población del Banco de Seguros del Estado existe un evento traumático desencadenante del cuadro clínico: el accidente laboral. De cualquier forma, en todos los pacientes con dolor crónico, la historia traumática de abandono, maltrato o abuso sexual está presente en alta frecuencia.

Es importante el diagnóstico del trastorno de estrés postraumático y su vinculación con el dolor crónico ${ }^{(16)}$.

La incidencia del beneficio secundario del síntoma como perpetuador del mismo debe ser evaluada.

Como entidades psiquiátricas ampliamente estudiadas y descritas en su vinculación con el dolor crónico se encuentran la ansiedad y la depresión, que requieren diagnóstico y tratamiento adecuado.

Todos los aspectos hasta acá analizados tienen interferencia con el descanso nocturno, lo cual agrava la situación clínica, siendo un objetivo terapéutico mejorar el sueño.

La insatisfacción laboral, los litigios, las demandas físicas del trabajo, los incentivos financieros y el tiempo fuera del trabajo por enfermedad obstaculizan la rehabilitación del paciente.

Existen factores asistenciales que favorecen la perpetuación del síntoma: la indicación de exámenes paraclínicos en forma excesiva, la demora en obtener la consulta, los mensajes contradictorios de los distintos profesionales, la realización de procedimientos invasivos en forma excesiva e intempestiva, son aspectos que contribuyen a la perpetuación del sufrimiento.

En la figura 3 se muestra lo observado en la población asistida. Se destaca el diagnóstico clínico de depresión en $40 \%$, de trastorno de ansiedad y la alteración del sueño en más de la mitad, y la presencia de estrés postraumático en un número importante de pacientes. Casi 


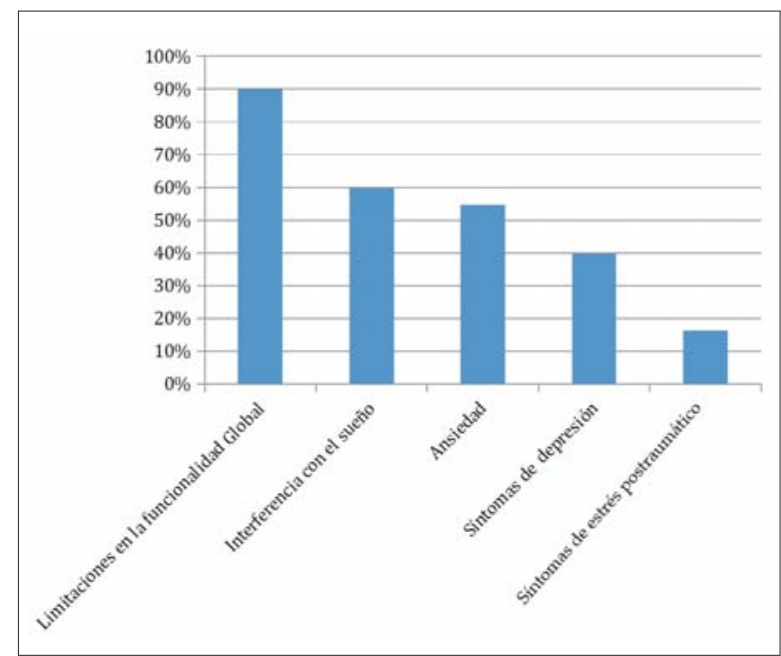

Figura 3. Diagnósticos asociados al dolor. Se destaca el diagnóstico clínico de depresión en el $40 \%$, de trastorno de ansiedad y la alteración del sueño en más de la mitad, y la presencia de estrés postraumático en un número importante de pacientes. Casi la totalidad presenta limitaciones en la funcionalidad global. Estos datos son concordantes con lo que se refiere en la literatura internacional.

la totalidad presentan interferencia para las actividades de la vida diaria. Estos datos son concordantes con lo que se refiere en la literatura internacional.

\section{Intervención clínico-terapéutica realizada en la población asistida}

La consulta con el equipo interdisciplinario tiene una finalidad diagnóstica y terapéutica, siendo fundamental el primer encuentro con el paciente. El abordaje empático, comprensivo y continente del paciente como una persona que sufre es fundamental, lo que tiene una incidencia positiva en la construcción del vínculo terapéutico y la alianza terapéutica.

El plan terapéutico es una construcción única para ese paciente que es único, dirigido a mejorar a la persona. Está planteado desde una perspectiva biopsicosocial, donde integramos los aspectos farmacológicos, psicológicos, familiares, sociales y de rehabilitación física.

No se trata de una suma de tratamientos, sino de un tratamiento integral, diseñado para el paciente, resultado de la evaluación clínica realizada, abordando todas las dimensiones involucradas con el objetivo de la rehabilitación y el reintegro al trabajo.

En cuanto al tratamiento farmacológico ${ }^{(17)}$, con cada indicación se explican los efectos que buscamos, las posibles reacciones adversas y cómo contrarrestarlas en el caso de que aparezcan. Se utilizaron analgésicos menores o mayores de acuerdo a la intensidad del dolor y

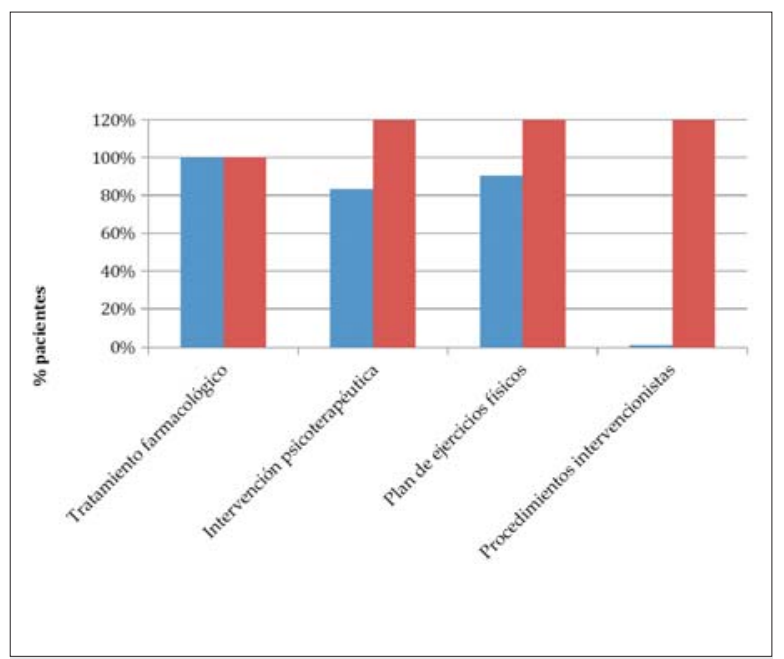

Figura 4. Intervenciones terapéuticas.

coadyuvantes según el tipo de dolor, si asociaban o no insomnio, ansiedad o depresión u otro trastorno ${ }^{(18)}$.

En cuanto a la rehabilitación física en el tratamiento del dolor crónico las terapias activas son las que han demostrado tener mayores beneficios a largo plazo ${ }^{(19)}$, por lo cual nuestro equipo promueve el movimiento. El papel del entrenamiento de las habilidades motoras, en contraste con la asistencia pasiva del ejercicio en general, ofrece mayor potencial para el éxito de la rehabilitación, asociado a mejoras en el rendimiento de la tarea debido a los cambios producidos en la reorganización cortical $^{(20)}$. A la mayoría de los pacientes se les confeccionó un plan de ejercicio activo individualizado, buscando la autonomía y el rol activo, intentando descentrar del sitio de dolor.

Desde la perspectiva psicológica y psiquiátrica el abordaje terapéutico incluye tanto el uso de fármacos como de intervenciones psicoterapéuticas. El tratamiento farmacológico de la ansiedad y la depresión es fundamental. Mejorar la calidad del sueño es un objetivo a conseguir siempre. Las intervenciones psicológicas son realizadas desde diferentes marcos teóricos, adecuados a las necesidades concretas de cada paciente. En este sentido utilizamos recursos técnicos desde la perspectiva cognitiva, sistémica y psicoanalítica. Muchas veces, corregir creencias sobre la enfermedad o los tratamientos es un objetivo en sí mismo. Otras veces puede ser intervenir para mejorar aspectos vinculares familiares o sociales, creando redes de soporte. Desde la perspectiva psicoanalítica utilizamos fundamentalmente intervenciones de apoyo como señalamiento, clarificación y confrontación. En algunas situaciones específicas hemos derivado a algunos pacientes para un abordaje psicoterapéutico específico en policlínica con psicólogo de 
la institución, abordaje complementario al nuestro y planificado en conjunto.

Con respecto a los tratamientos intervencionistas queremos resaltar que muchos de los pacientes tratados en la unidad habían recibido tratamientos invasivos previos: 32 pacientes habían sido intervenidos quirúrgicamente en una o más oportunidades, 33 habían recibido bloqueos anestésicos también en una o más oportunidades.

Nuestro equipo entiende estos tratamientos en un conjunto de medidas terapéuticas. Debemos evitar la retraumatización que significa la realización de procedimientos invasivos si no hay una clara indicación o evidencia de probable beneficio (figura 4).

\section{Consideraciones finales}

De lo analizado hasta ahora podemos concluir que estamos frente a un problema clínico extremadamente complejo que exige un enorme esfuerzo de conceptualización desde diferentes perspectivas teóricas y en el cual aún no existe ninguna evidencia demostrada. A la altura actual del conocimiento, podemos plantear como certeza que el abordaje integral e interdisciplinario es el adecuado, que el mismo apunta a la mejora global del paciente y no solo al alivio del dolor.

Para finalizar, pensamos que el paciente con dolor crónico se comporta generalmente como un paciente con una enfermedad crónica. Frecuentemente requerirá mantenerse vinculado a los equipos asistenciales. En este sentido, el concepto de alta requiere ser revisado y contextualizado en relación con la situación del paciente. Son pacientes que requerirán apoyos farmacológicos, de rehabilitación física, de intervenciones psicoterapéuticas en forma permanente y secuencial, con reevaluaciones periódicas. La vinculación del paciente con el equipo interdisciplinario especializado en dolor crónico puede variar en el tiempo, pudiendo ser referido al primer nivel de atención para su seguimiento, sin que esto implique una desvinculación total. El objetivo central es mejorar la calidad de vida, optimizar sus vínculos familiares y sociales, y mantener una inserción laboral adecuada, impidiendo que el síntoma se transforme en el centro de su vida.

\section{Abstract}

Chronic pain is a global public health problem, and according to estimations, it affects around $20 \%$ of the population. Evidence is telling us the best assistance model for this kind of patients is through multidisciplinary teams, focusing on communication among the different disciplines and building up a common approach to patients.

The Pain Multidisciplinary Unit at Maciel Hospital National Insurance Bank involves the implementation of a biopsychosocial assistance model for Chronic Pain, whose therapeutic goal is the comprehensive rehabilitation of the patient. This study describes the population assisted in the first seven months of operation at the $\mathrm{Na}-$ tional Insurance Bank, the comprehensive evaluation conducted by the team and the most relevant results, as well as the definition of a personalized therapeutic plan.

Chronic low back pain was the most frequent clinical condition. Upon comprehensive assessment we point out how pain interfered with basic everyday life activities in $90 \%$ of patients, sleep alterations in $60 \%$ and depression diagnosed on $40 \%$ of the population assisted. The studies provides a detailed account of interventions around the three therapeutic pillars: pharmacologic, physical rehabilitation and psychotherapy, having the main objective been defined as the individual's rehabilitation and going back to work.

\section{Resumo}

A Dor Crônica é um problema de Saúde Pública em todo o mundo; estima-se que afeta ao redor de $20 \%$ da população. Cada vez mais há evidências de que o modelo de atenção para pacientes com Dor Crônica seja composto por equipes interdisciplinares, com ênfase na comunicação entre as diferentes disciplinas, e na construção de uma forma conjunta de ver o paciente.

A "Unidad Interdisciplinaria de Dolor del Hospital Maciel - Banco de Seguros del Estado" é o resultado de colocar em prática um modelo biopsicossocial de atenção à Dor Crônica, no qual o objetivo terapêutico é a reabilitação integral da pessoa. Neste artigo descreve-se a população atendida nos primeiros 7 meses de trabalho no Banco de Seguros del Estado, a avaliação integral que a equipe realiza e os resultados mais relevantes, e também a construção do plano terapêutico individualizado.

A Lombalgia Crônica pós-cirurgia foi o quadro clínico mais frequente. Destaca-se da avaliação integral a Interferência da Dor com as Atividades Básicas da Vida Diária em $90 \%$, alterações do sono em $60 \%$ e o diagnóstico de Depressão em $40 \%$ da população atendida. As intervenções realizadas nos três pilares terapêuticos: farmacológico, reabilitação física e abordagem psicoterapêutica são descritas detalhadamente com o objetivo definido de reabilitar a pessoa e possibilitar sua reintegração ao trabalho.

\section{Bibliografía}

1. Breivik H, Collett B, Ventafridda V, Cohen R, Gallacher D. Survey of chronic pain in Europe: prevalence, impact on daily life, and treatment. Eur J Pain 2006; 10(4):287-333. 
2. Sociedad Española del Dolor. La prevalencia en España del dolor crónico podría llegar al 11\%. Diario Médico 2016.

3. Mencías Hurtado AB, Rodríguez Hernández JL. Trastornos del sueño en el paciente con dolor crónico. Rev Soc Esp Dolor 2012; 19(6):332-4.

4. López-Silva MC, Sánchez de Enciso M, Rodríguez-Fernández MC, Vázquez-Seijas E. Cavidol: calidad de vida y dolor en atención primaria. Rev Soc Esp Dolor 2007; 14(1):9-19.

5. International Association for the Study of Pain. Task Force on Taxonomy. Merskey H, ed. Classification of chronic pain: descriptions of chronic pain syndromes and definitions of pain terms. 2nd. ed. Seattle: IASP, 1994.

6. Montes MJ, Retamoso I, Vázquez C. El Dolor: un abordaje interdisciplinario. Montevideo: Zona Editorial, 2012.

7. Kamper SJ, Apeldoorn AT, Chiarotto A, Smeets RJ, Ostelo RW, Guzman J, et al. Multidisciplinary biopsychosocial rehabilitation for chronic low back pain: Cochrane systematic review and meta-analysis. BMJ 2015; 350:h444.

8. Stolkiner A. La interdisciplina: entre la epistemología y las prácticas. Campo Psi 1999. Disponible en: http://www.campopsi.com.ar/ [Consulta: 5 de mayo 2018].

9. Solitario RC, Garbus P, Stolkiner A. Atención Primaria de la salud e interdisciplina: dos componentes claves para las reformas en salud mental. Rev Asoc Med Bahía Blanca 2007; 17(3):63-8.

10. De la Herrán A. Complejidad y transdisciplinariedad. Rev Educação Skepsis 2011; 2: 294-320.

11. González Baroìn $\mathbf{S}$, Rodríguez Loìpez $\mathbf{M}$. El dolor: fisiopatologiìa. Clì̀nica. Sistemas de Medicioìn. En: González Baroìn $\mathrm{S}$, Rodríguez Loìpez $\mathrm{M}$, dirs. Dolor y caìncer: hacia una oncología sin dolor. Madrid: Médica Panamericana, 2003:7-32.

12. Moyá F, Grau M, Riesco N, Núñez M, Brancós MA, Valdés M, et al. Dolor lumbar crónico. Valoración multidisciplinaria de 100 pacientes. Aten Prim 2000; 26(4):239-44.
13. Haythornthwaite JA. Evaluación de las creencias sobre el dolor, de las estrategias de afrontamiento y del funcionalismo en la vida diaria. En: McMahon S, Koltzenburg M, eds. Wall y Melzack- Tratado del dolor. Madrid: Elsevier, 2006:321-34.

14. RuehIman B, Karoly P, Pugliese J. Psychosocial correlates of chronic pain and depression in young adults: further evidence of the utility of the Profile of Chronic Pain: Screen (PCP: S) and the Profile of Chronic Pain: Extended Assessment (PCP: EA) battery. Pain Med 2010; 11:1546-53.

15. Wong WS, Chen PP, Yap J, Mak KH, Tam BK, Fielding R. Chronic pain and psychiatric morbidity: a comparison between patients attending specialist orthopedics clinic and multidisciplinary pain clinic. Pain Med 2011; 12:246-59.

16. Siqveland J, Ruud T, Hauff E. Post-traumatic stress disorder moderates the relationship between trauma exposure and chronic pain. Eur J Psychotraumat 2017; 8(1):1375337.

17. Kroenke K, Krebs EE, Bair MJ. Pharmacotherapy of chronic pain: a synthesis of recommendations from systematic reviews. Gen Hosp Psychiatry 2009; 31:206-19.

18. Turk DC, Wilson HD, Cahana A. Treatment of chronic non-cancer pain. Lancet 2011; 377(9784):2226-35.

19. Geneen LJ, Moore RA, Clarke C, Martin D, Colvin LA, Smith BH. Physical activity and exercise for chronic pain in adults: an overview of Cochrane Reviews. Cochrane Database Syst Rev 2017. Disponible en: www.cochranelibrary.com [Consulta: 10 julio 2018].

20. Boudreau S, Farina D, Falla D. The role of motor learning and neuroplasticity in designing rehabilitation approaches for musculoskeletal pain disorders. Man Ther 2010; 15(5):410-4 Disponible en: www.elsevier.com/math [Consulta: 10 julio 2018]. 